\title{
Transcriptome analysis identified genes involved in anthocyanin biosynthesis in Rainbow bamboo (Indosasa hispida MeClure cv. 'Rainbow')
}

\author{
Yi Wang, YuMing Yang, Juan Wang ${ }^{*}$, Yuan XiaoLong,
}

Key Laboratory of Yunnan Provincial for Forest Plant Cultivation and Utilization, and Key Laboratory of the State Forestry Administration for Conservation of Yunnan Rare, Endangered \& Endemic Plant, Yunnan Academy of Forestry, Kunming650204, China

\section{*Corresponding author: schima@163.com}

\begin{abstract}
Rainbow bamboo (Indosasa hispida) is an ornamental plant, which contains unique red to purple anthocyanin in its culm. However, the biosynthesis and function of anthocyanin in bamboo remains unclear. In this study, RNA-seq was used to investigate the transcriptome of the species and compare the gene expression profiles of red and white culms. The expression levels of genes involved in the anthocyanin biosynthesis pathway were detected using quantitative reverse transcription-polymerase chain reaction (qRT-PCR). In total, 5.92 billion reads were obtained from the culm of Rainbow bamboo, which were assembled into 60,716 unigenes. qRT-PCR showed that the expression levels of anthocyanin biosynthesis-related genes in the red and white culms were higher than that in green leaves and that their levels in the red culm without sheath were higher than that in the white culm with sheath. Transcriptome analysis and qRT-PCR showed that the differences in the expression of genes encoding chalcone isomerase (CHI), dihydroflavonol reductase (DFR), flavonoid 3'-hydroxylase (F3' $\mathrm{H})$, and anthocyanidin 3-O-glycosyltransferase (A3GT) between the culm and leaf were significant. This implies that CHI, DFR, F3' $\mathrm{H}$, and A3GT play important roles in anthocyanin synthesis and accumulation in the culm of Rainbow bamboo.
\end{abstract}

Keywords: Indosasa hispida, Anthocyanin biosynthesis, Transcriptome analysis, High-throughput sequencing, qRT-PCR Abbreviations:PAL_phenylalanine ammonialyase; $\mathrm{C} 4 \mathrm{H}$ _cinnamate 4-hydroxylase; 4CL_4-coumarate CoA ligase; CHS_chalcone synthase; CHI_chalcone isomerase; $\mathrm{F} 3 \mathrm{H}$ _flavanone 3-hydroxylase; $\mathrm{F} 3^{\prime} \mathrm{H}_{-}$flavonoid $3^{\prime}$-hydroxylase; $\mathrm{F3}^{\prime} \mathrm{5}^{\prime} \mathrm{H}_{-} \quad$ flavonoid 3',5'-hydroxylase; FLS_flavonol synthase, DFR_4-reductase gene; ANS_anthocyanidin synthase; A3GT_3-O-glucosyltransferase gene; A5GT_anthocyanidin 5-O-glucosyltransferase gene; MT_methyl transferase gene.

\section{Introduction}

Bamboo belongs to subfamily Bambusoideae, family Gramineae, and is distributed in the subtropics and tropics of Asia, Africa, and Latin America. Bamboo is an important forest type of southern China and a horticultural plant in China. The culm of bamboo is usually green; however, Rainbow bamboo (Indosasa hispida McClure cv. Rainbow) possesses red and purple culm, which contributes to its ornamental value and market potential (Wang et al., 2012). Studies show that anthocyanin is the red pigment in the culm of Rainbow bamboo (Zhang and Shi, 2015). However, the mechanism of anthocyanin biosynthesis in bamboo is still unclear. Anthocyanins, with water-soluble pigments of red, purple, and blue colors, occur in flowers, fruits, and leaves of plants. In plants, anthocyanin usually attracts pollinators and seed dispersers. Recent studies showed that anthocyanin also acts against abiotic stresses such as oxidative stress, UV (ultraviolet)-B radiation, visible light, and temperature variation, and biotic stress factors such as pathogens, insects, and herbivores (Manetas et al., 2006; Hatier et al., 2008; Wang et al., 2013). Anthocyanin biosynthesis occurs in three steps in model plants (Zhang et al., 2014). Formation of
Coumarate-CoA is the first step, which involves enzymes such as phenylalanine ammonia lyase (PAL), cinnamate 4-hydroxylase $(\mathrm{C} 4 \mathrm{H})$, and 4-coumarate $\mathrm{CoA}$ ligase (4CL). Anthocyanidin is formed in the next step, involving the activities of chalcone synthase (CHS), chalcone isomerase (CHI), flavanone 3-hydroxylase $(\mathrm{F} 3 \mathrm{H})$, flavonoid $3^{\prime}$-hydroxylase $\left(\mathrm{F}^{\prime} \mathrm{H}\right)$, flavonoid $3^{\prime}, 5^{\prime}$-hydroxylase $\left(\mathrm{F}^{\prime} \mathrm{S}^{\prime} \mathrm{H}\right)$, and flavonol synthase (FLS). Various anthocyanidins are formed in the final step via dihydroflavonol 4-reductase (DFR), anthocyanidin synthase (ANS), and modified enzymes such as anthocyanidin 3-O-glucosyltransferase (A3GT), anthocyanidin 5-O-glucosyltransferase (A5GT), and methyl transferase (MT). Although anthocyanin biosynthesis has been characterized in many plants (Zhao et al., 2014; Wei et al., 2015; Zhang et al., 2016; Li et al., 2018; Cao et al., 2018), reports regarding the mechanism of anthocyanin biosynthesis in bamboo are absent. In this study, the transcriptomes of the white and red culms of Rainbow bamboo were analyzed, the genes involved in anthocyanin biosynthesis were identified, and their functions were characterized. 


\section{Results}

\section{Sequence analysis and assembly of transcriptomes}

To examine the molecular basis of anthocyanin biosynthesis in the red culm of Rainbow bamboo, the red culm and green leaf were used to construct two libraries using high-throughput sequencing Illumina Hiseq 2500. The two libraries (red culm and white culm) produced 2.57 million and 68.65 million reads of raw data, respectively, from paired-end reads, with a single read length of $\sim 126$ bp (NCBI accessions: SRR4014063). After removal of adaptor sequences, ambiguous reads, and low-quality reads $(Q 20<20), 7,257,358,674$ and 7,438,594,324 nucleotides of high-quality clean reads were obtained from the two libraries, respectively. After assembly of all high-quality reads, 60,716 unigenes were obtained from red culm and leaf, and their N50 is 1500 (Fig 1).

\section{Functional annotation of transcriptome}

Unigenes in the transcriptome were annotated using several databases, namely, Ensembl plant (http://plants.ensembl.org/index.html), National Center for Biotechnology Information (http://www.ncbi.nlm.nih.gov/), EggNOG (http://eggnogdb.embl.de/), and Kyoto Encyclopedia of Genes and genomes (KEGG) (http://www.genome.jp/kegg/). The unigene sequences were first aligned to protein databases such as Swiss-Prot, Clusters of Orthologous Groups (COG,) and NR (E-value $<0.00001$ ) using BLASTx, and to the nucleotide database NT (E-value < 0.00001) using BLASTn, retrieving proteins with the highest sequence similarity to the identified unigenes along with their protein function annotations. In total, 49,751 unigenes were annotated using all databases, and only 15,520 unigenes were annotated in COG proteins database. These unigenes were distributed among 25 functional categories (Fig. 2).

\section{Transcriptome-based analysis of differentially expressed genes}

A rigorous algorithm was used to identify genes that were differentially expressed between the red culm and green leaf of Rainbow bamboo. The expression of 1,219 unigenes in leaf was higher than that in the red culm, whereas the expression of 2,376 unigenes in the culm was higher than that in the leaf. The unigenes with no significant differences were marked in red, whereas those with significant differences marked in blue (Fig. 3).

Differentially expressed genes were also analyzed for pathway enrichment, specifically biochemical and signal transduction pathways. Three flavonoid biosynthesis pathway enzymes were differentially expressed, namely, $\mathrm{CHI}, \mathrm{F}^{\prime} \mathrm{H}$, and DFR (Fig. 4). Analysis of the differentially expressed genes in the anthocyanin biosynthesis pathway showed that the expression of A3GT in the red culm genotype was higher than that in the leaf (Fig. 5). CHI (EC 5.5.1.6) is one of the key enzymes in the flavonoid biosynthesis pathway, catalyzing the stereospecific isomerization of chalcones into (2S)-naringenin (Morita et al. 2014). Four $\mathrm{CHI}$ unigenes were selected from the transcriptome database. After ORF and conserved domain analysis, two complete $\mathrm{CHI}$ genes (c73764_g1_i1 and c74095_g3_i1) were identified in Rainbow bamboo. Comparison of the expression of c73764_g1_i1 and c74095_g3_i1 based on transcriptome data showed that the expression of c73764_g1_i1 in the red culm was higher than that in the leaf. $\mathrm{F}^{\prime} \mathrm{H}$ (EC 1.14.13.21) transforms dihydroflavonol into dihydroquercetin by adding a hydroxyl to the 3' position of ring B (Schwinn et al., 2014). The transcriptome data indicated the existence of five $\mathrm{F}^{\prime} \mathrm{H}$-encoding genes. Other genes in the transcriptome data that are involved in anthocyanin biosynthesis included four DFR unigenes and five A3GT unigenes.

\section{Gene expression profile of anthocyanin biosynthesis in $I$. hispida}

Next, we attempted to confirm that the unigenes identified using sequencing and computational analysis were indeed expressed, and also analyzed the differences in gene expression profile between white culm (young culm with sheath), red culm, and green leaf. Nine unigenes related to anthocyanin synthesis were selected for qRT-PCR analysis, while glyceraldehyde-3-phosphate dehydrogenase (GAPDH) was used as the reference gene (Fig. 6). Gene expression in the red culm was higher than that in the leaf. However, the expression levels of $\mathrm{CHI}$ and DFR in the red culm were considerably higher than that in the leaf, and their ratios were 449 and 558, respectively. The expression level of $F 3^{\prime} H$ in the red culm was higher in the leaf, with a ratio of 41 . Gene expression in the red culm was slightly higher than that in the white culm. However, the expression of anthocyanidin synthase in white culm was higher than that in red culm. This indicates that anthocyanin in Rainbow bamboo was synthesized in both white and red culm, and anthocyanin accumulated in the red culm. Genes related to anthocyanin synthesis showed background expression in leaves. Therefore, leaves do not possess any visible anthocyanin.

\section{Discussion}

Bamboo is an important non-timber forest product, which is mainly distributed in the tropical and sub-tropical regions of the world. Bamboo is also a popular horticultural plant in China. However, breeding of bamboo is challenging because of long flowering cycle of up to 120 years, seed sterility, and low seed viability (Singh et al., 2013). Therefore, genetic engineering can be used to modify and breed new cultivars of bamboo. In particular, several research groups have successfully introduced foreign genes into bamboo cells using Agrobacterium tumefaciens binary vector or particle bombardment-mediated transformation (Wiersma, 2008; Ogita et al., 2011; Qiao et al., 2014; Sood et al., 2014). Therefore, breeding of new genetically engineered bamboo cultivars will be feasible in the future.

Next-generation sequencing technology has immensely promoted molecular biological research on bamboo. The first draft of the bamboo genome sequence was completed in 2013 (Peng et al., 2013a). Compared to genome sequencing, RNA sequencing (RNA-Seq) is a simpler and more efficient tool for obtaining abundant information regarding gene expression, gene regulation, and biosynthetic pathways (Wang et al., 2009; Ozsolak et al., 2011). Therefore, RNA sequencing will provide comprehensive information regarding plant metabolites and physiological processes (Kalra et al., 2013). In particular, de novo transcriptome sequencing in non-model organisms has emerged as a powerful and cost-effective tool. 
Table 1. primers used in qRT-PCR.

\begin{tabular}{lll}
\hline Primer name & Sequence (5'-3') & Detected gene name \\
\hline CHS-F & GTGCGACAAGTCCATGATCC & Chalcone synthase \\
CHI-F & GTGGTGCAGAAGACGAGGTG & \\
CHI-R & CTCACCGTTGCGTTCTCCAA & Chalcone isomerase \\
F3'H-F & GAAACTGACACGGGCTCCAC & \\
F3'H-R & ACGAGTCCAACAGCTACAAGG & Flavonoid 3'-monooxygenase \\
DFR-F & CCGTCGAACTGGTTGTGGAT & \\
DFR-R & AGGACAACTGGAGCGACATC & Dihydroflavonol reductase \\
ANR-F & GCCTCGTTCCCTGTGATGAG & \\
ANR-R & ACGGGTACATAGCTTCAGCG & Anthocyanidin reductase \\
ANS-F & TCTCTGCCTTGACGTTCACC & \\
ANS-R & GGGTTCAGGGCTCATTCGAT & Anthocyanidin synthase \\
A5,3GT-F & CGTCATCTTCGGCTCAGTCC & Anthocyanidin \\
A5,3GT-R & ACGTTGTCTTCATCCCGAGC & 5,3-O-glucosyltransferase \\
A3GT-F & GTTCACGACGGAGATGTCAA & anthocyanidin \\
A3GT-R & GGTAAGTGGGGAAGTAGGCG & 3-O-glycosyltransferase \\
A3G6"RhaT-F & CGACGGCCTTGCTGCGAGGA & Anthocyanidin 3-O-glucoside \\
A3G6"RhaT-R & GTGAACGCCGAGCAGTGGAC & 6"-O-rhamnosyltransferase \\
GAPDH-F & GCCGATGTTTGTGGTGGTG & Glyceraldehyde-3-phosphate \\
GAPDH-R & CCAGTCCTTGCTTGAGGGTC & dehydrogenase \\
\hline
\end{tabular}

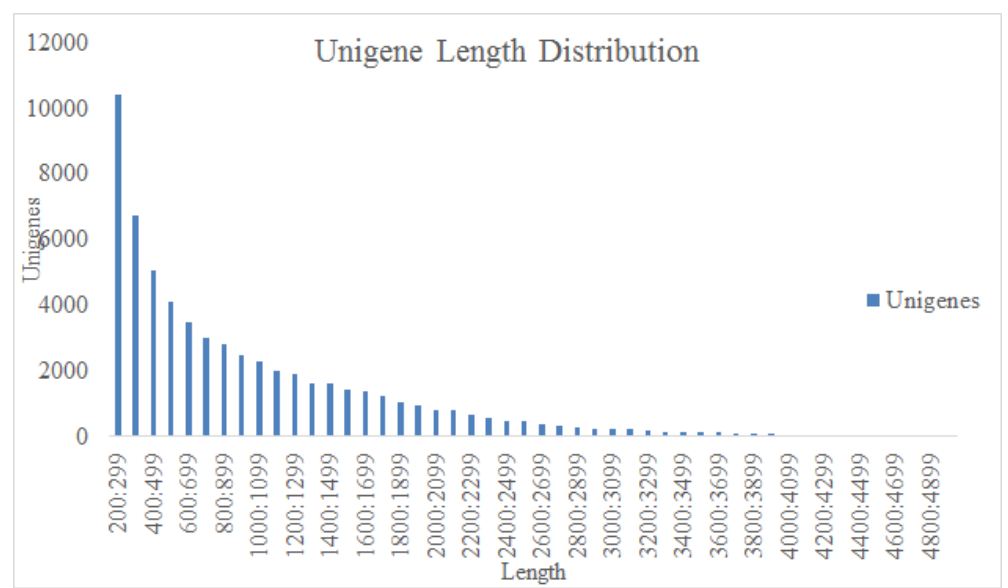

Fig 1. The length distribution of unigenes, the horizontal values are unigene lengths and the vertical values are numbers of unigenes.

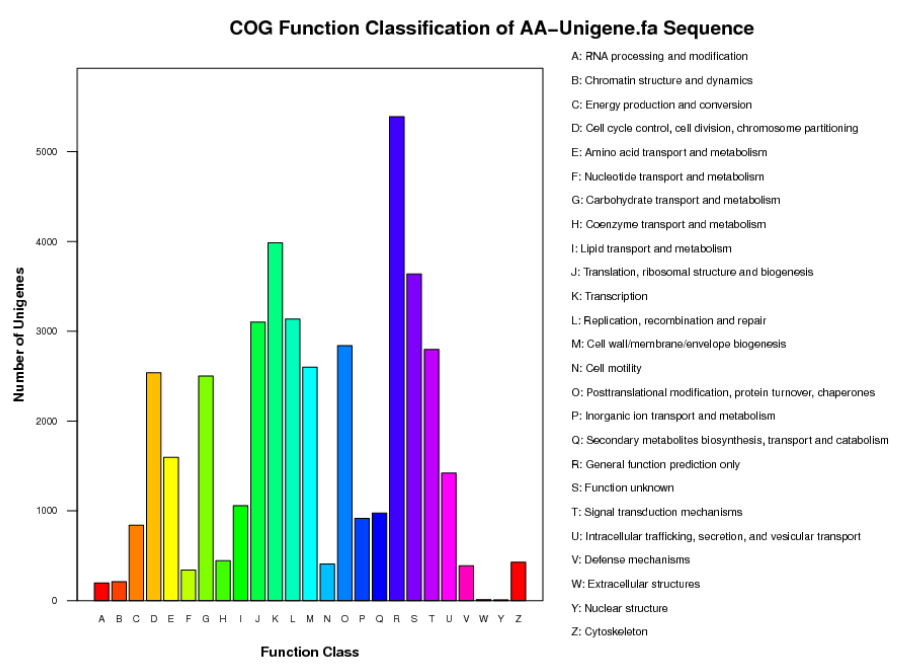

Fig 2. COG function classification of Unigenes in All-Unigene. The horizontal coordinates are function classes of cog, and the vertical coordinates are numbers of Unigenes in one class. The notation on the right is the full name of the functions in X-axis. 


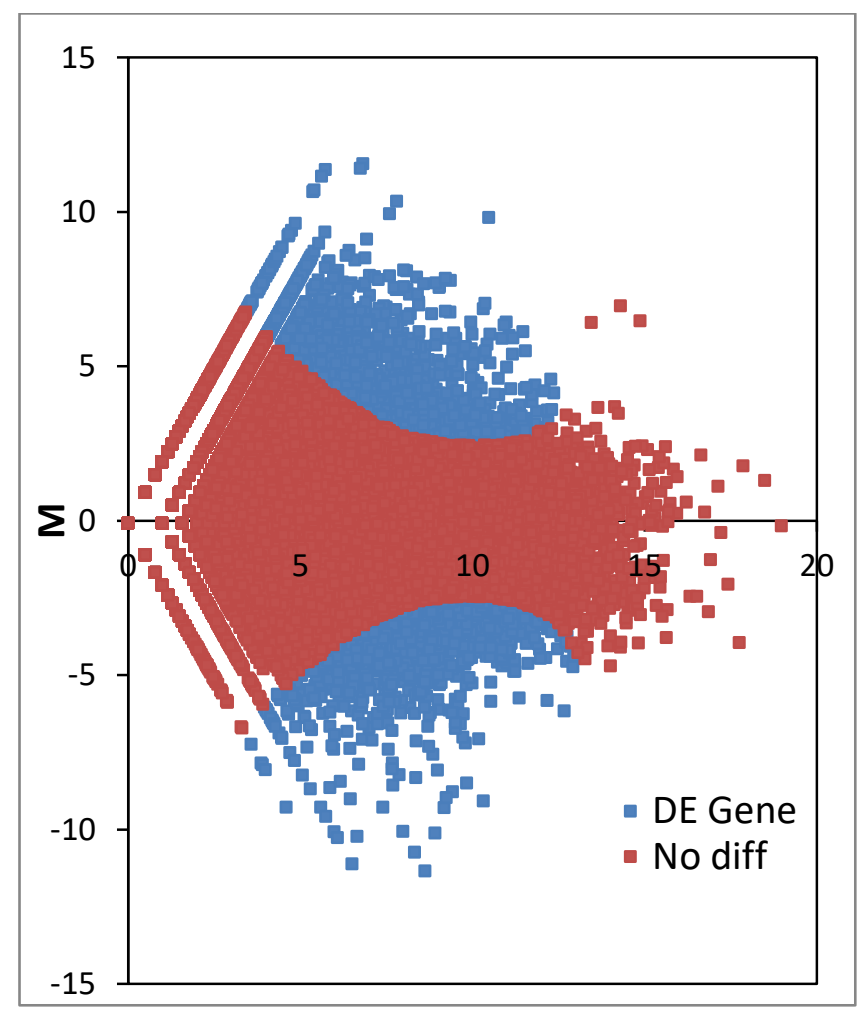

Fig 3. Expression level of culm vs leaf of Rainbow bamboo. The Red colour showed the gene with no significant different expression in culm and leaf. The blue colour showed gene with different expression in culm and leaf. The number of horizontal axis showed gene expression intensity, and the number of vertical axis showed expression difference.

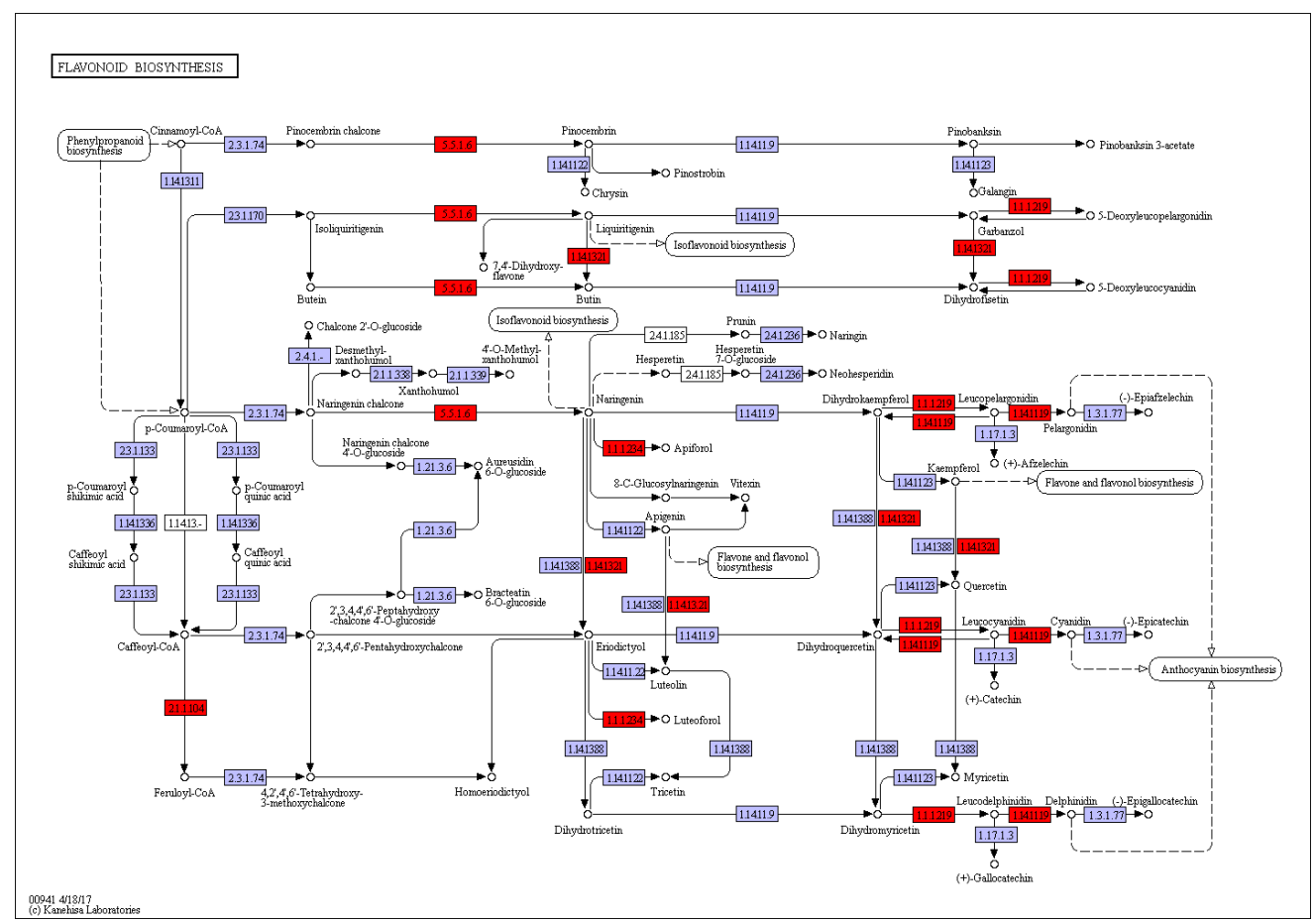

Fig 4. Differentially expressed genes significantly enriched in flavonoid biosynthesis. Red box showed the gene expression in culm was higher than in leaf. Blue box showed the gene expression is no significant difference between culm and leaf. White box showed the gene was not detected in transcriptome. 


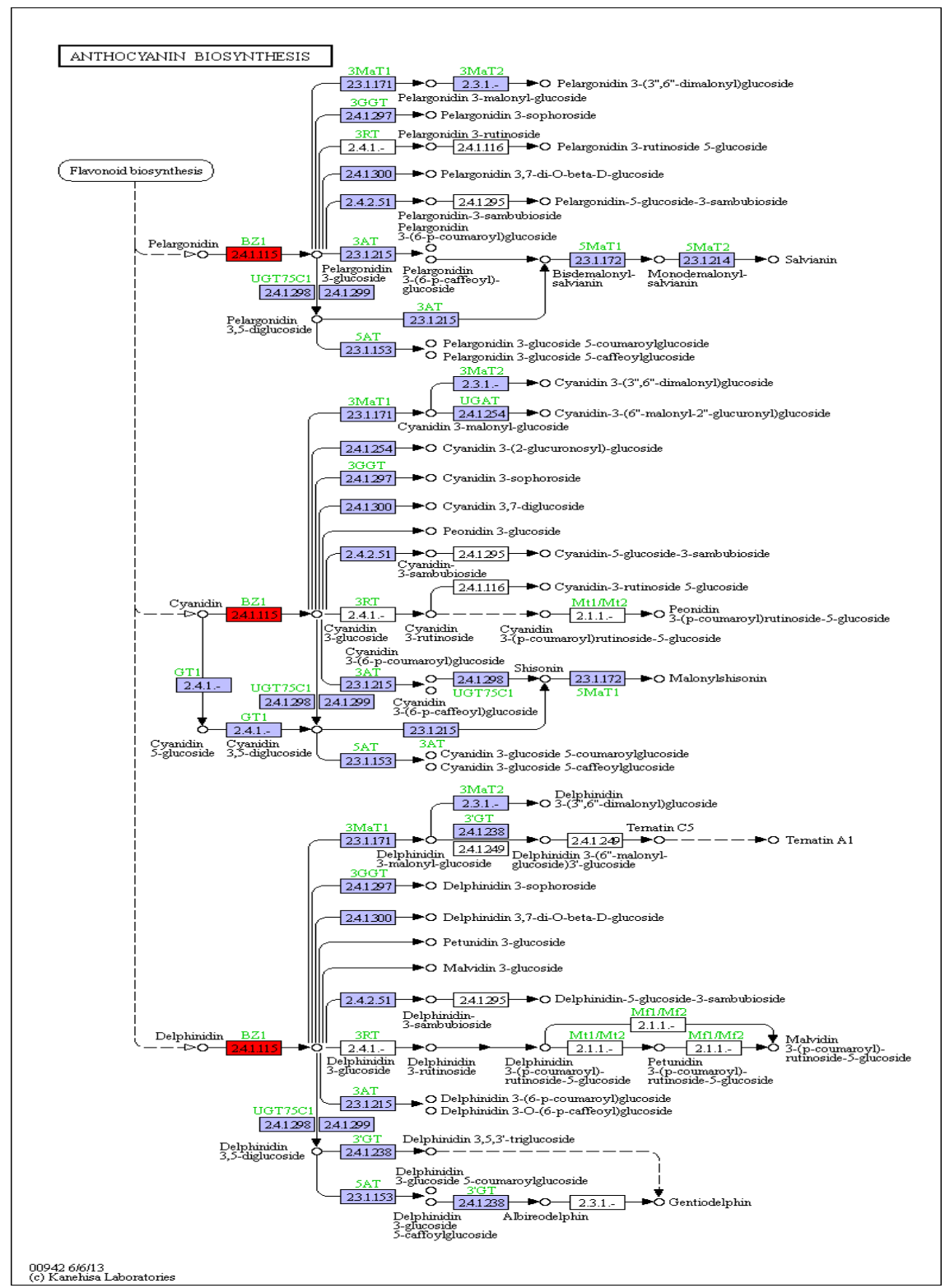

Fig 5. Differentially expressed genes significantly enriched in anthocyanin biosynthesis. Red box showed the gene expression in culm was higher than in leaf. Blue box showed the gene expression is no significant difference between culm and leaf. White box showed the gene was not detected in transcriptome.

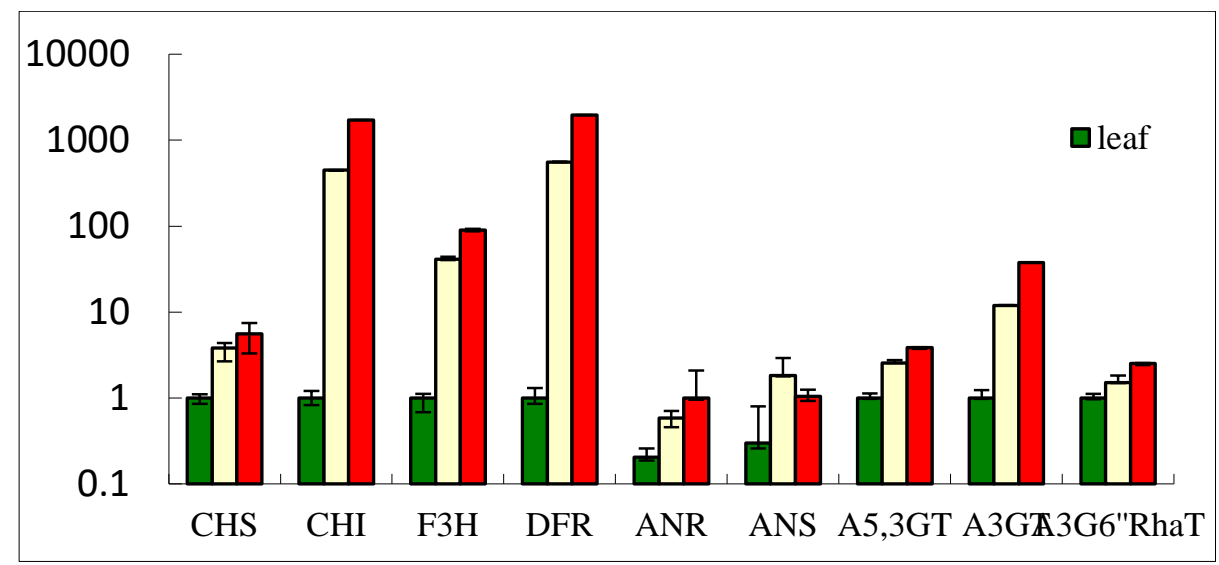

Fig 6. qRT-PCR analysis of 9 flavonoid biosynthetic pathway-related candidate unigenes in red stem, white stem and leaf of Rainbow bamboo. CHS: chalcone synthase; $\mathrm{CHI}$ : chalcone isomerase; F3H: flavonoid 3'-hydroxylase; DFR: dihydroflavonol reductase; ANR: anthocyanidin reductase; ANS: anthocyanidin synthase; A5,3GT: anthocyanidin 5,3-0-glucosyltransferase; A3GT: anthocyanidin 3-O-glycosyltransferase; A3G6"RhaT: anthocyanidin 3-O-glucoside 6"-O-rhamnosyltransferase. 
Till date, several transcriptome analyses of bamboo have been completed, which have revealed information related to fast growth and floral development (Liu et al., 2012; Zhang et al., 2012; He et al., 2013; Peng et al., 2013b; Gao et al., 2014). In this study, RNA-sequencing was applied to understand the mechanism of anthocyanin biosynthesis in Rainbow bamboo. Anthocyanins are ubiquitous in higher plants. However, in lower plants such as green algae and bryophytes, the anthocyanin biosynthetic enzymes were not detected (Dinabandhu, 2014). In addition, bryophytes (mosses) do not contain anthocyanins, but possess chalcones, flavonols, and flavones (Harashima, 2004). Therefore, anthocyanin synthesis is believed to be associated with adaptive evolution of terrestrial plants. Anthocyanins in flowers, seeds, and fruits are used for attracting or repelling pollinators and seed dispersers. The possible functions of anthocyanins in leaves, stems, roots, and other vegetative organs have long attracted scientific debate. The key functions of anthocyanins in vegetative organs are possibly protect chloroplasts from the adverse effects of excess light, attenuation of UV-B radiation, and antioxidant activity (Hatier et al., 2008).

Rainbow bamboo is new cultivar of I. hispida. The leaf is green with slight white bands, the culm without sheath has red color, whereas the culm with sheath is creamy white. The qRT-PCR showed that the expression levels of anthocyanin biosynthesis-related genes in the red and white culm were higher than those in the green leaf, and the expression in red culm without sheath was higher than that in the white culm with sheath. This implied that the anthocyanin biosynthesis-related genes were expressed in tissue-specific manner in Rainbow bamboo, and that the UV (ultraviolet)-B radiation and visible light intensifies anthocyanin production in the culm of Rainbow bamboo. Therefore, anthocyanin in Rainbow bamboo possibly attenuates UV-B radiation and visible light. Analysis of the differentially expressed genes using transcriptomics and qRT-PCR showed that genes encoding $\mathrm{CHI}$, dihydroflavonol reductase, $\mathrm{F}^{\prime} \mathrm{H}$, and anthocyanidin 3-O-glycosyltransferase are key regulators of anthocyanin biosynthesis in Rainbow bamboo. It is possible that $\mathrm{CHI}$ is key enzyme that regulates the flux in the anthocyanidin synthesis pathway in the culm of Rainbow bamboo.

\section{Materials and methods}

\section{Plant materials}

The red culm, white culm covered with sheath, and green leaf of Rainbow bamboo were collected in July 2014 at the Yunnan Academy of Forestry Arboretum, Kunming, China. All samples were immediately frozen in liquid nitrogen and stored at $-80^{\circ} \mathrm{C}$ for RNA extraction.

\section{RNA extraction, library construction, and RNA-Seq}

Total RNA was isolated using a RNeasy plant mini kit (Qiagen, Hilden, Germany), The RNA concentration and quality were examined using a NanoDrop 2000 spectrophotometer (Thermo Scientific, Waltham, MA, USA) and $0.8 \%$ agarose gel electrophoresis, respectively. Library construction and RNA-Seq were performed by the Personal Biotechnology Co. Ltd. (Shanghai, China). The TruSeq RNA sample preparation kit (Illumina) was used for purification and fragmentation of
mRNA. The first strand CDNA was synthesized using reverse transcriptase and the cleaved RNA as template, and the second-strand cDNA was synthesized using DNA polymerase I. The ends of the DNA fragments were modified and ligated with adapters, and the cleaned ligation products were used as template for PCR to enrich the products. The CDNA library was obtained, and the quality was assessed using the PicoGreen assay kit and Agilent 2100 Bioanalyzer (Santa Clara, CA, USA). The constructed cDNA libraries were sequenced on the Illumina HiSeq 2500 instrument in Shanghai Personal Biotechnology Co. Ltd.

\section{De novo transcriptome assembly and annotation}

Following the sequencing of the cDNA library, the high-quality clean reads were generated by trimming the raw reads to remove adapter sequences, low-quality reads with Q-values < 20 , or ambiguous bases (' $N$ '). The clean reads were then assembled de novo using the Trinity platform (http://trinityrnaseq.sf.net.). All unigenes were used as queries in Nr, EggNOG, Gene Ontology (GO), KO, and Swissprot databases (E-value $<10^{-5}$ ) and functionally annotated using GO analysis with the Blast2GO software $\left(\mathrm{E}-\right.$ value $\left.<10^{-5}\right)$. Metabolic pathways were predicted by KEGG mapping.

\section{GO classification of differentially expressed unigenes and pathway analysis}

To compare the expression abundance between the red clum and green leaf of Rainbow bamboo, unigene expression was calculated using the fragments per kb per million reads (FPKM) method. All high-quality reads were aligned to the assembled transcripts, and their tags were normalized into reads per kilobase of transcript per million mapped reads (RPKM) values. The differentially expressed genes between the two samples were identified using a rigorous algorithm based on the Audic and Claverie (1997) method. All differentially expressed genes were mapped to each term of the GO database (http://www.geneontology.org/), and the number of genes associated with each GO term was determined. The names and numbers of genes for each GO term were listed, and then the hypergeometric test was used to determine significantly enriched GO terms in differentially expressed genes compared to the genomic background. All differentially expressed genes were also mapped onto the KEGG pathway, with Q-value $\leq$ 0.05 considered to be significantly enriched in differentially expressed genes.

\section{qRT-PCR}

Nine genes involved in anthocyanin biosynthesis pathways were identified from the transcriptome of Rainbow bamboo databases. They included chalcone synthase (CHS, accession number: AJB28732); chalcone isomerase ( $\mathrm{CHI}$, accession number: AJB28731); flavonoid 3'-hydroxylase (F3H, accession number: KJ784467); dihydroflavonol reductase (DFR, accession number: AHD25273; anthocyanidin reductase (ANR, accession number: KJ784465); anthocyanidin synthase (ANS, accession number: KF268002); anthocyanidin 5,3-O-glucosyltransferase (A5,3GT, accession number: KX819304); anthocyanidin 3-O-glycosyltransferase (A3GT, accession number: AJB28730); anthocyanidin 3-0-glucoside 6"-O-rhamnosyltransferase (A3G6"RhaT, accession number: KX819305). The expression 
profiles were assayed using qRT-PCR. First, special serial primers were designed for the nine genes (Table 1). The cDNA was synthesized using $1 \mu \mathrm{g}$ total RNA each from red culm, white culm, and green leaf using a reverse transcriptase kit (TaKaRa Super RT Kit, Dalian, China) according to the manufacturer's instruction. The SYBR Green was used for detecting PCR products using the StepOnePlus real-time PCR system (Applied Biosystems, Foster City, CA, USA). GAPDH was used as the internal control for normalization of gene expression. At least two independent biological replicates and three technical replicates of each biological replicate for each sample were analyzed using qRT-PCR to ensure reproducibility and reliability.

\section{Conclusion}

In this study, we performed a comprehensive transcriptomic analysis of red culm and green leaf samples from Rainbow bamboo, which provided a valuable resource for genetic and genomic study in the future. Analysis of differentially expressed genes using transcriptomics and qRT-PCR of red culm, white culm, and leaf showed that the genes in the anthocyanin biosynthesis pathway were differentially expressed. The differences in the expression levels of $\mathrm{CHI}$, DFR, F3' $H$, and A3GT in the culm and leaf were especially significant. This implies that $\mathrm{CHI}, \mathrm{DFR}, \mathrm{F} 3$ 'H and A3GT play important roles in anthocyanidin synthesis and accumulation in the culm of Rainbow bamboo. Overall, our work provides new information regarding the molecular mechanisms of anthocyanin biosynthesis in Rainbow bamboo.

\section{Acknowledgments}

The study was supported by grants from the Science and Technology Planning Project of Yunnan Province (2013FA054, 2014IA013, 2015IA005), the Middle Aged Academic and Technical Leader Project of Yunnan Province (Grant 2010Cl016), and the Yunnan Academy of Forestry Innovation Fund Project, Grant No. QN2018-01.

\section{References}

Audic S, Claverie JM (1997) The significance of digital gene expression profiles. Genome Res. 7:986-995.

Cao K, Ding TY, Mao DM, Zhu GR, Fang WC, Chen CW, Wang XW, Wang LS (2018) Transcriptome analysis reveals novel genes involved in anthocyanin biosynthesis in the flesh of peach. Plant Physiol Bioch. 123:94-102.

Dinabandhu A (2014) Computational and evolutionary insights into anthocyanin biosynthesis genes between Solanaceae and Poaceae. Int J Bioinform Biol Sci. 2:165-177.

Gao J. Zhang Y, Zhang C, Qi F, Li X, Mu S, Peng Z (2014) Characterization of the floral transcriptome of moso bamboo (Phyllostachys edulis) at different flowering developmental stages by transcriptome sequencing and RNA-seq analysis. PloS One. 6: e98910.

Harashima S, Takano H, Ono K, Takio S (2004) Chalcone synthase-like gene in the liverwort, Marchantia paleacea var. diptera. Plant Cell Rep. 23:167-173.

Hatier JHB, Gould KS (2008) Anthocyanin function in vegetative organs in: anthocyanins. Springer, New York, NY
He CY, Cui K, Zhang JG, Duan AG, Zeng YF (2013) Next-generation sequencing-based $\mathrm{mRNA}$ and microRNA expression profiling analysis revealed pathways involved in the rapid growth of developing culms in moso bamboo. BMC Plant Biol. 13:1-14.

Kalra S, Puniya BL, Kulshreshtha D, Kumar S, Kaur J, Ramachandran S (2013) De novo transcriptome sequencing reveals important molecular networks and metabolic pathways of the plant, Chlorophytum borivilianum. PLoS One. 8: e83336.

Li YZ, Luo X, Wu CY, Cao SY, Zhou YF, Jie B, Cao YL, Meng HJ, Wu GL (2018) Comparative transcriptome analysis of genes involved in anthocyanin biosynthesis in red and green walnut (Juglans regia L.). Molecules. 23:25.

Liu MY, Qiao GR, Jiang J, Yang HQ, Xie LH, Xie JZ, Zhou RY (2012 Transcriptome sequencing and de novo analysis for ma bamboo (Dendrocalamus latiflorus Munro) using the illumina platform. PLoS One. 7: e46766.

Manetas Y (2006) Why some leaves are anthocyanic and why most anthocyanic leaves are red? Flora. 201:163-177.

Morita Y, Takagi K, Fukuchi MM, Ishiguro K, Tanaka Y, Nitasaka E, Nakayama M, Saito N, Kagami T, Hoshino A, lida S (2014) A chalcone isomerase-like protein enhances flavonoid production and flower pigmentation. Plant J. 78:294-304.

Ogita S, Kikuchi N, Nomura T, Kato Y (2011) A practical protocol for particle bombardment-mediated transformation of Phyllostachys bamboo suspension cells. Plant Biotechnol. 28:43-50.

Ozsolak F, Milos P (2011) RNA sequencing: advances, challenges and opportunities. Nat Rev Genet. 12:87-98.

Peng ZH, Lu Y, Li LB, Zhao Q, Feng Q, Gao ZM, Lu HY, Hu T, Yao N, Liu KY, Li Y, Fan DL, Guo YL, Li WJ, Lu YQ, Weng QJ, Zhou CC, Zhang L, Huang T, Zhao Y, Zhu CR, Liu XG, Yang XW, Wang T, Miao K, Zhuang CY, Cao XL, Tang WL, Liu GS, Liu YL, Chen J, Liu ZJ, Yuan LC, Liu ZH, Huang XH, Lu TT, Fei BH, Ning ZM, Han $B$, Jiang ZH (2013a) The draft genome of the fast-growing non-timber forest species moso bamboo (Phyllostachys heterocycla). Nat Genet. 45:456-461.

Peng ZH, Zhang CL, Zhang Y, Hu T, Mu SH, Li XP, Gao J (2013b) Transcriptome sequencing and analysis of the fast growing shoots of moso bamboo (Phyllostachys edulis). PLoS One. 8:e78944.

Qiao GR, Yang HQ, Zhang L, Han XJ, Liu MY, Jiang J, Jiang YC, Zhou RY (2014) Enhanced cold stress tolerance of transgenic Dendrocalamus latiflorus munro (ma bamboo) plants expressing a bacterial $\operatorname{cod} A$ gene. In Vitro Cell Dev Biol Plant. 50:385-391.

Schwinn K, Miosic S, Davies K, Thill J, Gotame TP, Stich K, Halbwirth H (2014) The B-ring hydroxylation pattern of anthocyanins can be determined through activity of the flavonoid 3'-hydroxylase on leucoanthocyanidins. Planta. 240:1003-1010.

Singh SR, Singh R, Kalia S, Dalal S, Dhawan AK, Kalia RK (2013) Limitations, progress and prospects of application of biotechnological tools in improvement of bamboo-a plant with extraordinary qualities. Physiol Mol Biol Plants. 19:21-41.

Sood P, Bhattacharya A, Joshi R, Gulati A, Chanda S, Sood A (2014) A method to overcome the waxy surface, cell wall thickening, and polyphenol induced necrosis at wound sites-the major deterrents to Agrobacterium mediated transformation of bamboo, a woody monocot. J Plant Biochem Biotechnol. 23:69-80. 
Wang J, Sun H, Peng GS, Wang MY, Xiong Z, Zhang XB, Sun MS, Yang YM (2012) Phylogenetic study on anthocyanin produced mutant of Indosasa hispida. J West China Fore Sci. 41:1-6.

Wang HX, Fan WJ, Li H, Yang J, Huang JR, Zhang P (2013) Functional characterization of dihydroflavonol-4-reductase in anthocyanin biosynthesis of purple sweet potato underlies the direct evidence of anthocyanins function against abiotic stresses. PLoS One. 8: e78484.

Wang Z, Gerstein M, Snyder M (2009) RNA-seq: a revolutionary tool for transcriptomics. Nat Rev Genet. 10:57-63.

Wei HR, Chen X, Zong XJ, Shu HR, Gao DS, Liu QZ (2015) Comparative transcriptome analysis of genes involved in anthocyanin biosynthesis in the red and yellow fruits of sweet cherry (Prunus avium L.). PLoS One. 10: e0121164.

Wiersma R (2008) Bioluminescent bamboo. Newsl South Calif Chap Am Bamboo Soc. 18:2-5.
Zhang, CS, Shi R (2015) Determination of anthocyanins content in different parts of Indosasa hispida McClure cv.'Rainbow'. Int J Sci. 2:82-85.

Zhang HN, Li WC, Wang HC, Shi SY, Shu B, Liu LQ, Wei YZ, Xie JH (2016) Transcriptome profiling of light-regulated anthocyanin biosynthesis in the pericarp of litchi. Front Plant Sci. 7:963.

Zhang XM, Zhao L, Larson-Rabin Z, Li DZ, Guo ZH (2012) De novo sequencing and characterization of the floral transcriptome of Dendrocalamus latiflorus (Poaceae: Bambusoideae). PLoS One. 7:e4282.

Zhang Y, Butelli E, Martin C (2014) Engineering anthocyanin biosynthesis in plants. Curr Opin Plant Biol. 19:81-90.

Zhao DQ, Jiang Y, Ning CL, Meng JS, Lin SS, Ding W, Tao J (2014) Transcriptome sequencing of a chimaera reveals coordinated expression of anthocyanin biosynthetic genes mediating yellow formation in herbaceous peony (Paeonia lactiflora Pall.). BMC Genomics. 15: 689. 